\title{
Mediastinoscopy-assisted transhiatal esophagectomy versus thoraco-laparoscopic esophagectomy for esophageal cancer: a single-center initial experience
}

\author{
Wenyi Liu ${ }^{1}$, Xiaotong Guo ${ }^{1}$, Hongbo Zhao ${ }^{1}$, Xin Yu ${ }^{1}$, Chunguang Wang ${ }^{1}$, Longde Du ${ }^{1}$, Feng Wang ${ }^{1}$, \\ Juwei $\mathrm{Mu}^{1,2}$ \\ ${ }^{1}$ Department of Thoracic Surgery, National Cancer Center/National Clinical Research Center for Cancer/Cancer Hospital \& Shenzhen Hospital, \\ Chinese Academy of Medical Sciences and Peking Union Medical College, Shenzhen, China; ${ }^{2}$ Department of Thoracic Surgery, National Cancer \\ Center/National Clinical Research Center for Cancer/Cancer Hospital, Chinese Academy of Medical Sciences and Peking Union Medical College, \\ Beijing, China \\ Contributions: (I) Conception and design: W Liu; (II) Administrative support: None; (III) Provision of study materials or patients: W Liu; (IV) \\ Collection and assembly of data: W Liu; (V) Data analysis and interpretation: All authors; (VI) Manuscript writing: All authors; (VII) Final approval \\ of manuscript: All authors. \\ Correspondence to: Juwei Mu. Department of Thoracic Surgery, National Cancer Center/National Clinical Research Center for Cancer/Cancer Hospital, \\ Chinese Academy of Medical Sciences and Peking Union Medical College, Beijing, China. Email: mujuwei@cicams.ac.cn; mujuwei@chcamssz.ac.cn.
}

Background: We aimed to compare mediastinoscopy-assisted transhiatal esophagectomy (MATHE) with thoraco-laparoscopic esophagectomy (TLE) for patients with esophageal cancer in terms of the clinical effectiveness and perioperative complications.

Methods: In total, 98 patients who underwent esophagectomy consecutively for esophageal squamous cell carcinoma in our center from Jan. 2018 to Dec. 2019 were included in this study. Thirty patients underwent mediastinoscopy-assisted and laparoscopic transhiatal esophagectomy with cervical anastomosis (the MATHE group). The other sixty-eight patients received TLE (the TLE group). Each patient's general conditions and perioperative complications were recorded.

Results: Patients in the MATHE group were observed to have a higher incidence of postoperative hoarseness than those in the TLE group. There were no significant differences between the MATHE group and the TLE group in regards to the operation time, intraoperative blood loss, number of lymph nodes dissected or postoperative hospital stay. Similarly, no statistically significant differences were observed in the incidence of anastomotic fistula, respiratory complications, or chylothorax or in the conversion rate or inhospital mortality rate between the two groups.

Conclusions: The short-term efficacy in the MATHE group was similar to that in the TLE group, although patients in the MATHE group may have had a higher incidence of postoperative hoarseness. Therefore, MATHE may be a feasible and safe surgical procedure for appropriate patients with esophageal cancer.

Keywords: Mediastinoscopy; transhiatal esophagectomy; thoraco-laparoscopic esophagectomy (TLE); esophageal cancer

Submitted Mar 16, 2020. Accepted for publication Jul 18, 2020.

doi: $10.21037 /$ jtd-20-1328

View this article at: http://dx.doi.org/10.21037/jtd-20-1328 


\section{Introduction}

Esophageal cancer is a major disease that seriously threatens the health and life of Chinese people. According to cancer statistics from 2015, the incidence of esophageal cancer in China has risen to a rank of fourth among all malignant tumors, and the mortality rate ranks seventh (1). At present, surgery is still the most important and effective treatment for esophageal cancer and has been an irreplaceable treatment procedure $(2,3)$.

The anatomical features of the esophagus fundamentally determine the diversity of surgical approaches and methods. The esophagus is located in the posterior mediastinum and crosses the neck, chest and abdomen longitudinally. The left thoracic, right thoracic and mediastinal approaches are the three main surgical approaches for esophageal cancer. The classic left thoracic approach includes conventional left thoracotomy esophagectomy and a transthoracic combined left cervical incision. The right thoracic approach includes two incisions (right posterolateral thoracic incision + midupper abdominal incision) and three incisions (left neck incision + right posterolateral thoracic incision + midupper abdominal incision) as well as thoracoscopic and laparoscopic minimally invasive two- or three-incision esophagectomy. There are several techniques available for esophageal cancer. When esophageal tumours have a high location, the McKeown approach is needed.

Thoraco-laparoscopic esophagectomy (TLE) has been applied for more than 30 years, and its clinical efficacy, safety and tumor curativeness have been proven by experts. This procedure is mainly divided into three steps: the first step is thoracoscopic esophageal mobilization and lymph node dissection; the second step is laparoscopic gastric dissection and lymph node dissection; and the third step is gastroesophageal neck anastomosis or gastroesophageal intrathoracic anastomosis without neck incision. With the advanced technological development of minimally invasive endoscopic techniques, mediastinoscopyassisted transhiatal esophagectomy (MATHE) is being applied for the surgical treatment of esophageal cancer. This surgical approach combines mediastinoscopy with laparoscopy and mainly includes the following two steps: thoracic esophagus mobilization and lymphadenectomy under mediastinoscopy alongside laparoscopic gastric dissection and abdominal lymph node dissection; and gastroesophageal neck anastomosis. In 2015, the Japanese scholar Hitoshi Fujiwara systematically reported a method of cervical mediastinoscopy combined with laparoscopic esophagectomy; since then, this procedure has been gradually applied worldwide $(4,5)$. Some retrospective studies have shown that compared to TLE, MATHE further reduces surgical trauma, and it seems to be more advantageous in protecting respiratory function (6-8). However, there are currently no multicenter randomized controlled studies comparing MATHE with TLE for esophageal cancer. The purpose of this study was to preliminarily explore the feasibility, safety, curative effect and recent clinical efficacy of MATHE for esophageal cancer compared with thoracoscopic laparoscopic esophageal cancer surgery. Through this comparative study, we provide initial experience with the advantages and disadvantages of MATHE and TLE, which will lay the foundation for our further prospective randomized controlled study. We present the following article in accordance with the STROBE reporting checklist (available at http://dx.doi.org/10.21037/jtd-20-1328).

\section{Methods}

The surgeons had rich experience in mediastinal lymph node biopsy and resection with traditional mediastinoscopy. After applying the MATHE as a new technology and new project in our hospital, a total of 8 MATHE procedures were performed in 2017. The patients included in this study were all recruited after 2018. All patients who received the procedure signed informed consent forms. This study is conducted in accordance with the principles of the Declaration of Helsinki (as revised in 2013).

\section{Patients}

In total, 98 patients who underwent esophagectomy consecutively for esophageal squamous cell carcinoma in the Cancer Hospital Chinese Academy of Medical Sciences, Shenzhen Center from Jan. 2018 to Dec. 2019 were included in this study. Thirty patients aged 40-79 years underwent mediastinoscopy-assisted and laparoscopic transhiatal esophagectomy with cervical anastomosis (the MATHE group). The other sixty-eight patients aged 40-79 years received TLE (the TLE group). Two patients in the MATHE group and ten patients in the TLE group received neoadjuvant therapy before surgery. There were no significant differences in terms of age, sex or other demographic parameters between the two groups (Table 1). 
Table 1 Patient characteristics

\begin{tabular}{lccc}
\hline Variables & MATHE $(\mathrm{n}=30)$ & TLE $(\mathrm{n}=68)$ & $\mathrm{P}$ \\
\hline Age (years) & $58.03 \pm 8.79$ & $56.97 \pm 8.88$ & 0.585 \\
Sex & & & 0.645 \\
Male & 24 & 57 & \\
Female & 6 & 11 & \\
Preoperative complications & & \\
Hypertension & 3 & 8 & 0.799 \\
Diabetes & 4 & 6 & 0.497 \\
Arrhythmia & 0 & 1 & 0.507 \\
Staging & & & 0.643 \\
cT (1/2/3/4) (\%) & $33.3 / 33.3 / 26.7 / 6.7$ & $17.6 / 44.1 / 29.4 / 8.8$ & \\
cN (0/1) (\%) & $53.3 / 46.7$ & $55.9 / 44.1$ & \\
$\begin{array}{l}\text { Neoadjuvant } \\
\text { therapy }\end{array}$ & 2 & 10 & 0.263 \\
\hline
\end{tabular}

MATHE, mediastinoscopy-assisted transhiatal esophagectomy;

TLE, thoraco-laparoscopic esophagectomy.

\section{Inclusion criteria}

Patients who met the following criteria were included in the study: (I) histologically diagnosed with squamous cell carcinoma by endoscopic biopsy, cT1-4N0-1M0; (II) aged between 18 and 80 years; (III) primary tumor is located in the thoracic esophagus; (IV) no clinical evidence of distant organ metastasis; and (V) adequate organ function. The preoperative clinical $\mathrm{T}$ stage of esophageal cancer was evaluated by endoscopic ultrasonography and chest enhanced CT, while the $\mathrm{N}$ and $\mathrm{M}$ stages were evaluated by cervical, chest, and abdominal enhanced CT, PET-CT, bone scan and MRI.

\section{Exclusion criteria}

Patients were excluded if they had one of the following: (I) cervical or abdominal esophageal carcinoma; (II) previous esophagectomy, gastrectomy, or mediastinal surgery; (III) current uncontrolled illness such as severe cardiac disease, uncontrollable hypertension or diabetes, or active bacterial infection; (IV) unable to tolerate tracheal intubation and general anesthesia as determined by an anesthesiologist preoperatively; (V) pregnant or lactating women; or (VI) ECOG PS score $>2$.

\section{Surgical procedures}

\section{MATHE}

Mediastinoscopy-assisted and laparoscopic transhiatal esophagectomy with cervical anastomosis (MATHE) was performed in the supine position by two teams simultaneously operating from the abdomen and neck.

The mediastinum, the space behind the sternum in the middle of the chest, is situated between the two lungs. It contains the heart and its great vessels and the trachea, esophagus, thymus gland and lymph nodes. This area can be examined with a tool called a mediastinoscope. The rationale of MATHE is to establish artificial pneumomediastinum and explore the mediastinal structures with a video-assisted procedure, through which esophageal dissociation and lymphadenectomy can be completed.

The neck team used a $5-\mathrm{mm}$ oblique scope for mediastinoscopy. A $4.5-\mathrm{cm}$ skin transverse incision was made $1.5-\mathrm{cm}$ above the left supraclavicular notch, and the left recurrent laryngeal nerve (RLN) was exposed and marked with a yellow band. The cervical esophagus was separated and marked with a blue band, and the lymph nodes in the above areas were dissected at the same time. A special incision protective sheath and trocar components were inserted, and artificial mediastinum inflation was adjusted with $\mathrm{CO}_{2}$ to an insufflation pressure of 6-8 $\mathrm{mmHg}$. Mediastinoscopy was used to remove the upper and middle thoracic esophagus from the top down to the lower level of the tracheal carina. Then, dissection of the lymph nodes around the left RLN, paraesophageal nerve and subcarina was performed.

The abdominal team completed the abdominal operation at the same time. There were five incisions: a $1.0-\mathrm{cm}$ paraumbilical incision was used as the observation hole; a second $1.0-\mathrm{cm}$ incision was placed under the xiphoid process; another $1.0-\mathrm{cm}$ incision was located at the junction of the right clavicular midline and $3-\mathrm{cm}$ horizontal above the umbilicus; and two $0.5-\mathrm{cm}$ incisions were located at the junction of the left clavicular midline and $3-\mathrm{cm}$ horizontal above the umbilicus and at the junction of the right clavicular midline and costal arch. After exploration of the abdominal cavity, the gastric collateral ligament, spleen and stomach ligaments, and short gastric blood vessels were separated from the spleen of the stomach by the outer edge of the right vascular arch of the gastric cardia along the direction of the greater curvature of the stomach. The lesser omentum was opened, the layer from the pancreatic capsule 
to the left portion of the stomach was separated, the left gastric blood vessels were fully exposed and ligated, and the lymph nodes belonging to the left gastric blood vessels were cleaned. The liver and stomach ligaments were displaced along the lesser curvature of the stomach and dissipated upward until the diaphragmatic foot was exposed. The lower thoracic esophagus was freed through the esophageal hiatus, and the lymph nodes along the side of the cardia and lower pulmonary vein were dissected. After completing the above operation, a 5 -cm vertical incision was made under the xiphoid process, and the stomach was pulled from the body to make a tubular stomach.

The esophagus was pulled from the left neck, and the mushroom-like portion was placed in the proximal portion of the esophagus. A portion of the xiphoid was excised, the posterior sternum space was opened, and the tubular stomach was lifted through the posterior sternum channel to the neck. Finally, the anastomosis was made with a stapler. Hand sewn anastomosis was also acceptable; nevertheless, it is not described in detail here.

\section{TLE}

The TLE procedure has been described previously (9).

Thoracoscopic phase: after successful anesthesia, the patient was placed in the left lateral decubitus position. Four thoracoscopic ports were established: first, a $1.0-\mathrm{cm}$ observation hole was established in the 7 th intercostal space of the right axillary line, and a thoracoscope was inserted to ensure that there were no adhesions or metastases in the thorax; another $1.0-\mathrm{cm}$ port was placed at the eighth or ninth intercostal space, posterior to the axillary line; and two $0.5-\mathrm{cm}$ ports were placed in the $3 \mathrm{rd}$ or 4 th intercostal space of the right anterior axillary line and the 7 th intercostal space of the right inferior scapular line. Artificial pneumothorax was established, and the $\mathrm{CO}_{2}$ pressure was set to $8-10 \mathrm{mmHg}$. The posterior mediastinal pleura was opened along the spinal column, fully freeing the upper thoracic esophagus, and the lymph nodes located in the upper right mediastinum and along the bilateral RLNs were removed. After severing the azygos arch, exposure of the lower thoracic esophagus was continued. After total lymphadenectomy and adequate hemostasis, a chest tube was routinely placed.

Laparoscopic phase: the patient was turned to the supine position, and a $15^{\circ}$ head-to-foot low supine position was achieved. The surgeon stood on the right side of the patient and placed a pneumoperitoneum needle under the umbilicus. A 1.0-cm hole (observation hole) was established for pneumoperitoneum, and the $\mathrm{CO}_{2}$ pressure was set to 12-14 mmHg. Then, another two $1.0-\mathrm{cm}$ holes and two $0.5-\mathrm{cm}$ holes were placed one by one. The abdominal cavity was examined to ensure that there was no metastasis. An ultrasonic knife was used to separate the omentum of the greater curvature of the stomach along the outer side of the vascular arch of the gastric omentum. The gastric and colonic ligaments, spleen and stomach ligaments, and short gastric vessels were separated in sequence. Attention was paid to protect the right vessels of the gastric omentum and the right vessels of the stomach. The left gastric vessel was fully exposed. The areas proximal and distal to the left gastric vessel were double-clamped with Hem-o-lock clips, and an ultrasonic knife was then used to sever the vessel. After total lymphadenectomy and adequate hemostasis, the stomach was pulled from the abdominal cavity to make a tubular stomach.

Cervical anastomosis: a 4-5-cm horizontal neck incision was made. The cervical esophagus was exposed, removed at the lower part, pulled into the abdominal cavity and prolapsed. Then, an incision was made along the stomach with a cutting suture to yield a tubular stomach with a width of approximately $3.5-\mathrm{cm}$. Finally, the tumor was removed. The prepared tubular stomach was pulled from the posterior mediastinum or the posterior sternal path to the neck. Finally, anastomosis was performed on the neck with a mechanical stapler or hand sewn in an end-to-side fashion.

\section{Postoperative care}

The patients were admitted to intensive care units (ICUs) or discharged to hospital wards directly after surgery. All patients received second-generation cephalosporins for prophylactic anti-infective treatment, conventional analgesic treatment with weak opioid or nonsteroidal analgesics, and the best nutritional support treatment (through intravenous or enteral nutrition) until the patient recovered to a semifluid diet and until routine treatment with atomization and expectoration was administered after the operation. If there was substantial blood drainage after the operation, hemostatic drugs were given properly. Assessment of hoarseness was undertaken on the first day postoperatively.

\section{Statistical analysis}

The patients' data were collected and analyzed using IBM SPSS Statistics for Windows, version 20 software 
Table 2 Short-term outcomes

\begin{tabular}{lccc}
\hline Variables & MATHE $(\mathrm{n}=30)$ & TLE $(\mathrm{n}=68)$ & $\mathrm{P}$ \\
\hline Operation time $(\mathrm{min})$ & $491.2 \pm 123.9$ & $484.1 \pm 118.0$ & 0.787 \\
Blood loss $(\mathrm{mL})$ & $256.0 \pm 120.0$ & $229.0 \pm 97.19$ & 0.241 \\
Lymph nodes dissected & $24.73 \pm 6.66$ & $27.97 \pm 9.12$ & 0.084 \\
Postoperative hospital & $33.50 \pm 23.25$ & $25.74 \pm 12.30$ & 0.093 \\
stay (days) & & & \\
Anastomotic fistula & 5 & 9 & 0.655 \\
Respiratory complications & 4 & 10 & 0.858 \\
Hoarseness & 8 & 5 & 0.009 \\
Chylothorax & 0 & 0 & $\mathrm{NS}$ \\
Conversion & 1 & 1 & 0.548 \\
In-hospital mortality & 0 & 1 & 0.504 \\
Distant metastasis & 1 & 1 & 0.548 \\
\hline
\end{tabular}

MATHE, mediastinoscopy-assisted transhiatal esophagectomy;

TLE, thoraco-laparoscopic esophagectomy; NS, not significant.

(IBM Corp., Armonk, NY, USA). Univariate analysis was performed by the Mann-Whitney $U$ test for continuous data and by the Pearson $\chi^{2}$ test for ordinal data. Fisher's exact test was used instead of the Pearson chi-square test if any expected cell value in a $2 \times 2$ table was less than five. $\mathrm{P}<0.05$ was considered significant.

\section{Results}

All patients in the two groups had successful surgeries. One patient in the MATHE group underwent conversion to thoracoscopic surgery because of severe adhesions between the tumor and the left main bronchus. One patient in the TLE group underwent conversion to thoracotomy due to the large tumor size, as the tumor was difficult to separate from the aorta by thoracoscopy. One patient in the TLE group died 2 months after the operation. The patient had a history of synchronous chemoradiotherapy before surgery. He died of sudden and massive hematemesis, which was thought to be caused by rupture and bleeding of the left common carotid artery.

Patients in the MATHE group had a higher incidence of postoperative hoarseness than those in the TLE group (8/30 vs. $5 / 68, \mathrm{P}<0.05)$. There were no significant differences between the MATHE group and TLE group in terms of the operation time $(491.2 \pm 123.9$ vs. $484.1 \pm 118.0, \mathrm{P}=0.787)$, intraoperative blood loss $(256.0 \pm 120.0$ vs. $229.0 \pm 97.19$,
$\mathrm{P}=0.241)$, number of lymph nodes dissected $(24.73 \pm 6.66$ vs. $27.97 \pm 9.12, \mathrm{P}=0.084$ ) or postoperative hospital stay (33.50 \pm 23.25 vs. $25.74 \pm 12.30, \mathrm{P}=0.093$ ). Similarly, no statistically significant differences were observed in the incidence of anastomotic fistula, respiratory complications, or chylothorax or in the conversion rate or in-hospital mortality rate between the two groups (Table 2).

The patient's vocal cord injury/palsy was assessed on the first day after the operation. A total of 13 patients had hoarseness: 8 in the MATHE group and 5 in the TLE group. They had changes in the sound of their voice, and they demonstrated the inability to raise their voice in volume. However, they had no difficulties swallowing or breathing, and they did not experience frequent choking while eating or drinking 2 weeks after surgery. According to the Clavien-Dindo (CD) and the Esophagectomy Complications Consensus Group (ECCG) classifications, all 13 patients had type I, vocal cord injury/palsy, which indicates a transient injury requiring no therapy (10).

Follow-up by telephone and outpatient reexamination revealed a median follow-up time of 11.2 months (ranging from 2 to 24 months). During the routine examination after the operation, 1 patient had liver metastasis in the TLE group. Furthermore, 1 patient had lung metastasis in the MATHE group.

\section{Discussion}

Compared with thoracic esophagectomy, mediastinal esophagectomy can ensure relative integrity of the thorax and has less interference with the heart and especially the lung. However, the feasibility and safety of resection for esophageal cancer through the mediastinum still requires more evidence $(11,12)$. Anatomically, important organs are located adjacent to the esophagus, such as the aorta, trachea, heart and RLN. It is theoretically easy to damage these important adjacent structures when operations are performed in the narrow space of the mediastinum. Once these structures are damaged, it is difficult to take remedial measures in a short time. Second, systematic lymph node dissection is particularly important in radical esophagectomy for esophageal cancer. Some scholars doubt that this method can achieve radical resection, which is inconsistent with the principles of surgical treatment for tumors (13). In particular, there are some technical difficulties in the dissection of lymph nodes of the right RLN and subcarinal lymph nodes. However, in recent years, studies by Japanese scholars have confirmed 
that lymph node dissection by mediastinoscopy can meet radical tumor requirements $(4,5)$. In our hospital, we pay special attention to the dissection of bilateral RLN lymph nodes and the dissection of subcarinal lymph nodes, which may also lead to a higher incidence of hoarseness. We reviewed the 30 patients who underwent MATHE, and the incidence of hoarseness was $33.3 \%(5 / 15)$ for the first 15 patients, and $20 \%$ (3/15) for the later 15 patients. This may indicate that improvements in surgical skills can reduce the incidence of vocal cord palsy, but there is a possibility of bias due to the small sample size of patients undergoing this surgery, and it is still necessary to explore whether there is a learning curve.

Compared with that of traditional transmediastinal esophagectomy, the innovation of pneumatic mediastinoscopy laparoscopic esophagectomy for esophageal cancer lies in the establishment of an artificial pneumatic mediastinum and the use of endoscopic visual systems and ultrasound scalpel and other micro-operating systems, which significantly enhance the real-time visibility and operability of mediastinal structures, thus improving the safety of surgery. Complete esophagectomy and mediastinal lymph node dissection are important factors affecting the survival rate of patients with esophageal cancer. With the help of the pneumatic mediastinum, surgeons can isolate and resect the esophagus and its surrounding tissues and lymph nodes. MATHE represents a less invasive surgical procedure, and this approach is worth investigating. Longterm outcomes and overall survival require further research for a clear answer $(14,15)$.

MATHE may be a feasible and safe surgical procedure for selected patients with esophageal cancer. However, the lack of conclusive evidence for better safety with MATHE, the limitations of preoperative $\mathrm{N}$-staging, and the satisfactory oncological outcomes of TLE, suggest that MATHE should only be adopted in a small minority of patients with esophageal cancer (13).

The preliminary experience of our center shows that there are still shortcomings with MATHE. The operation field in the mediastinum is smaller than that in the thorax, and the number of instruments inserted into the mediastinum is limited. The narrow operation space has a higher requirement for surgeons. Once bleeding occurs, conversion to thoracotomy is likely. There is still major difficulty with lymphadenectomy in terms of the deep mediastinal space around the aortic arch and tracheal bifurcation due to the narrow operation space and limited vision. Therefore, there was no significant difference between MATHE and TLE in terms of the number of lymph nodes dissected in our study. Furthermore, the interference of the RLN during the operation led to a higher incidence of hoarseness.

In conclusion, compared with that of TLE, the shortterm efficacy of MATHE is similar, although MATHE is associated with a higher incidence of postoperative hoarseness. However, the long-term outcomes and overall survival rates still require multicenter randomized controlled studies for validation.

\section{Acknowledgments}

Funding: This study was funded by National Cancer Centre Climbing Fund, National Key Research and Development Program (NCC201819B69), Bethune Charitable Foundation (HZB-20190528-12). The funders had no role in study design, data collection and analysis, decision to publish, or preparation of the manuscript.

\section{Footnote}

Reporting Checklist: The authors have completed the STROBE reporting checklist. Available at http://dx.doi. org/10.21037/jtd-20-1328

Data Sharing Statement: Available at http://dx.doi. org/10.21037/jtd-20-1328

Peer Review File: Available at http://dx.doi.org/10.21037/jtd20-1328

Conflicts of Interest: All authors have completed the ICMJE uniform disclosure form (available at http://dx.doi. org/10.21037/jtd-20-1328). The authors have no conflicts of interest to declare.

Ethical Statement: The authors are accountable for all aspects of the work in ensuring that questions related to the accuracy or integrity of any part of the work are appropriately investigated and resolved. The study conformed to the provisions of the Declaration of Helsinki (as revised in 2013). The present study was approved by the Ethics Review Committee of the National Cancer Center/ National Clinical Research Center for Cancer/Cancer Hospital \& Shenzhen Hospital. The number/ID of the Ethics Approval was 2020-16. All patients who received the procedure signed informed consent forms. 
Open Access Statement: This is an Open Access article distributed in accordance with the Creative Commons Attribution-NonCommercial-NoDerivs 4.0 International License (CC BY-NC-ND 4.0), which permits the noncommercial replication and distribution of the article with the strict proviso that no changes or edits are made and the original work is properly cited (including links to both the formal publication through the relevant DOI and the license). See: https://creativecommons.org/licenses/by-nc-nd/4.0/.

\section{References}

1. Chen W, Zheng R, Baade PD, et al. Cancer statistics in China, 2015. CA Cancer J Clin 2016;66:115-32.

2. Mao Y, He J, Gao S, et al. Controversies in the surgical treatment for esophageal carcinoma and future investigation. Zhonghua Wei Chang Wai Ke Za Zhi 2015;18:851-4.

3. Mu J, Yuan Z, Zhang B, et al. Comparative study of minimally invasive versus open esophagectomy for esophageal cancer in a single cancer center. Chin Med J (Engl) 2014;127:747-52.

4. Fujiwara H, Shiozaki A, Konishi H, et al. SinglePort Mediastinoscopic Lymphadenectomy Along the Left Recurrent Laryngeal Nerve. Ann Thorac Surg 2015;100:1115-7.

5. Fujiwara H, Shiozaki A, Konishi H, et al. Hand-assisted laparoscopic transhiatal esophagectomy with a systematic procedure for en bloc infracarinal lymph node dissection. Dis Esophagus 2016;29:131-8.

6. Jin Y, Lu X, Xue L, et al. Retrospective Comparison of Two Minimally Invasive Esophagectomy in the Treatment of Esophageal Cancer: Pneumatic Mediastinoscopy Versus Thoracoscopy. J Laparoendosc Adv Surg Tech A 2019;29:638-42.

7. Koide N, Takeuchi D, Suzuki A, et al. Mediastinoscopy-

Cite this article as: Liu W, Guo X, Zhao H, Yu X, Wang C, Du L, Wang F, Mu J. Mediastinoscopy-assisted transhiatal esophagectomy versus thoraco-laparoscopic esophagectomy for esophageal cancer: a single-center initial experience. J Thorac Dis 2020;12(9):4908-4914. doi: 10.21037/jtd-20-1328 assisted esophagectomy for esophageal cancer in patients with serious comorbidities. Surg Today 2012;42:127-34.

8. Feng MX, Wang H, Zhang Y, et al. Minimally invasive esophagectomy for esophageal squamous cell carcinoma: a case-control study of thoracoscope versus mediastinoscope assistance. Surg Endosc 2012;26:1573-8.

9. $\mathrm{Mu} \mathrm{J}$, Gao S, Mao Y, et al. Open three-stage transthoracic oesophagectomy versus minimally invasive thoracolaparoscopic oesophagectomy for oesophageal cancer: protocol for a multicentre prospective, open and parallel, randomised controlled trial. BMJ Open 2015;5:e08328.

10. Low DE, Alderson D, Cecconello I, et al. International Consensus on Standardization of Data Collection for Complications Associated With Esophagectomy: Esophagectomy Complications Consensus Group (ECCG). Ann Surg 2015;262:286-94.

11. Venissac N, Pop D, Mouroux J. Video-assisted mediastinoscopy as a therapeutic tool. Surg Endosc 2009;23:2466-72.

12. Bonavina L, Incarbone R, Bona D, et al. Esophagectomy via laparoscopy and transmediastinal endodissection. J Laparoendosc Adv Surg Tech A 2004;14:13-6.

13. Nomura T, Matsutani T, Hagiwara N, et al. Mediastinoscopy-assisted Transhiatal Esophagectomy for Esophageal Cancer: A Single-Institutional Cohort Study. Surg Laparosc Endosc Percutan Tech 2016;26:e153-e156.

14. Boshier PR, Anderson O, Hanna GB. Transthoracic versus transhiatal esophagectomy for the treatment of esophagogastric cancer: a meta-analysis. Ann Surg 2011;254:894-906.

15. Gockel I, Heckhoff S, Messow CM, et al. Transhiatal and transthoracic resection in adenocarcinoma of the esophagus: does the operative approach have an influence on the long-term prognosis? World J Surg Oncol 2005;3:40. 